\title{
Macrodinâmicas de Crescimento em uma Economia Solow-Swan com Migração: Uma Abordagem de Jogos Evolucionários ${ }^{\star}$
}

\author{
- JAYLSON JAIR DA SILVEIRA*
}

\begin{abstract}
RESUMO
Introduz-se no modelo Solow-Swan a migração como uma dinâmica satisficing. A conclusão geral está de acordo com a abordagem tradicional: as características da economia doméstica no estado estacionário dependem do saldo migratório líquido. Entretanto, novos resultados são obtidos. Em primeiro lugar, a economia doméstica não pode trilhar sua trajetória de crescimento equilibrado como uma perpétua receptora/fornecedora líquida de mão-de-obra. Em segundo lugar, caso ela apresente em seu estado estacionário um saldo migratório líquido nulo, o tamanho de sua população depende das condições iniciais (path dependence). Finalmente, demonstra-se que a introdução de um "efeito congestionamento" da imigração líquida abre a possibilidade de existência simultânea da economia doméstica e do resto do mundo.
\end{abstract}

Palavras-CHave

modelo Solow-Swan, migração, jogo evolucionário, dinâmica de seleção, dinâmica de satisficing

\begin{abstract}
Migration as a satisficing dynamics is introduced into the Solow-Swan model. The general conclusion is in agreement with that of the traditional approach: the steady-state features of domestic economy depends on the net migration rate. However, there are new outcomes. The domestic economy cannot be a perpetual host/supplier of migrants in its balanced growth path. If the domestic economy shows a zero-net migration rate in the steady state, then the population level will depend on the initial conditions (path dependence). A "congestion effect" resulting from net migration opens the possibility of simultaneous existence of the domestic economy and the rest of the world.
\end{abstract}

\section{KEY WORDS}

Solow-Swan model, migration, evolutionary game, selection dynamics, satisficing dynamics

\section{JEL CLASSIFICATION}

E32, J6I, O15, C79

\footnotetext{
+ Agradeço os comentários feitos pelo professor Renato Perim Colistete, bem com aqueles feitos, por ocasião da apresentação de uma versão prévia deste trabalho no Programa de Seminários Acadêmicos do IPE/USP, pelos professores Gilberto Tadeu Lima, Gustavo Gomes de Freitas e Jorge Eduardo de Castro Soromenho. Naturalmente, os erros remanescentes são de minha exclusiva responsabilidade.

* Professor do Departamento de Economia da FEA-RP/USP e membro do grupo de pesquisa COMPLEX - Economia e Complexidade/USP. Email: jaylson@usp.br. End. para contato: Av. Bandeirantes, 3900 - Monte Alegre - CEP 14040-900 - (16) 3602-3910 - Ribeirão Preto - SP.

(Recebido em abril de 2005. Aceito para publicação em agosto de 2006).
} 


\section{INTRODUÇÃO}

A migração é um processo resultante de inúmeras decisões, geralmente descentralizadas e não coordenadas. Tais decisões são tomadas em ambientes caracterizados por heterogeneidade dos agentes com relação às preferências de localização, aos custos de migração, ao conhecimento dos rendimentos e/ou amenidades das regiões de origem e/ou destino, às capacidades de coletar e processar as informaçóes disponíveis para prever eventos futuros etc. Entretanto, o processo migratório é comumente modelado como resultante de decisões de agentes homogêneos em um ambiente de racionalidade plena. Um exemplo desta abordagem é encontrado em Barro e Sala-i-Martin (2001, cap. 9, sec. 9.1), que analisam a interação entre migração internacional e o crescimento econômico de uma economia nacional. Estes autores modelam a decisão de migrar assumindo homogeneidade de custos de migração e de amenidades. Assumem também que os indivíduos são idênticos, otimizadores com horizontes infinitos e, dado o ambiente determinístico, capazes de calcular os valores presentes dos rendimentos futuros associados a cada localização possível (previsão perfeita).

O presente artigo também se propõe estudar a interação entre a migração internacional e o crescimento econômico de um país, todavia relaxando as hipóteses de homogeneidade e previsão perfeita. Supõe-se que os agentes tomam decisões sobre suas localizações sujeitas a limites cognitivos, como falta de pleno conhecimento dos rendimentos e/ou amenidades dos países de destino e de habilidade de prever perfeitamente os fluxos futuros de rendimentos e/ou amenidades das regiões de origem e destino. Para isso, toma-se como ponto de partida o modelo de crescimento econômico Solow-Swan e introduz-se em tal cenário o processo de migração que se desenrola em um ambiente de racionalidade limitada. O processo migratório será modelado como uma dinâmica evolucionária do tipo satisficing. ${ }^{1}$ Tal dinâmica será formalizada como um jogo evolucionário deduzido a partir da hipótese de que quanto mais baixo for o salário efetivo em uma economia, maior a proporção de indivíduos insatisfeitos que migram para outras economias em busca de maiores salários. ${ }^{2}$

Barro e Sala-i-Martin (2001, cap. 9, sec. 9.1) analisam o impacto do crescimento endógeno da força de trabalho sobre as propriedades da trajetória de crescimento equilibrado e da dinâmica de transição em uma economia Solow-Swan aberta à mi-

1 Segundo Simon (1987, p. 243), o próprio criador do termo, “... A decision maker who chooses the best available alternative according to some criterion is said to optimize; one who chooses an alternative that meets or exceeds specified criteria, but that is not guaranteed to be either unique or in any sense the best, is said to satisfice ...”

2 O presente artigo insere-se, do ponto de vista metodológico, num conjunto de trabalhos que tratam de questôes clássicas dentro da teoria econômica utilizando-se de uma abordagem da teoria dos jogos evolucionários. Entre tais trabalhos pode-se citar: Prado (1999); Prado (2001); Soromenho, Kadota e Prado (2001); Bonomo, Carrasco e Moreira (2003); Prado, Kadota e Soromenho (2003); Silveira (2003) e Silveira e Sanson (2003). 
gração. Tal análise é realizada a partir das premissas de que a população e a força de trabalho de uma economia mantêm uma relação de proporcionalidade estável e que as taxas naturais de crescimento das populações da economia doméstica e do resto do mundo são dadas e iguais. A conclusão central destes autores é que as características da economia doméstica no estado estacionário dependem do saldo migratório líquido. O presente artigo busca mostrar, tomando como referência a versão de Barro e Sala-i-Martin, que os resultados comumente alcançados relativos às propriedades do estado estacionário de uma economia Solow-Swan aberta à migração podem ser obtidos como propriedades emergentes da interação entre a dinâmica de acumulação de capital, como formalizada na tradição Solow-Swan, e a dinâmica migratória satisficing aqui proposta.

Além disso, novos resultados são obtidos. Em primeiro lugar, é demonstrado que economia doméstica não pode trilhar sua trajetória de crescimento equilibrado como uma perpétua receptora/fornecedora líquida de mão-de-obra. Em segundo lugar, caso a economia doméstica apresente em seu estado estacionário um saldo migratório líquido nulo, o tamanho de sua população depende das condições iniciais (path dependence). Finalmente, demonstra-se que a introdução de um "efeito congestionamento" da imigração líquida abre a possibilidade de existência simultânea da economia doméstica e do resto do mundo.

Este texto está organizado como segue. Na próxima seção apresenta-se um modelo de crescimento Solow-Swan com migração, desenvolvido por Barro e Sala-i-Martin (2001, cap. 9, sec. 1), o qual será utilizado como referência na avaliação dos resultados alcançados no modelo proposto no presente artigo. Na segunda seção deriva-se uma dinâmica de seleção que descreve o fluxo migratório entre a economia doméstica e o resto do mundo como uma dinâmica evolucionária do tipo satisficing. Na terceira seção estudam-se as características qualitativas do processo que emergem da interação entre a dinâmica de seleção associada ao fluxo migratório e a equação fundamental de crescimento Solow-Swan. Na quarta seção faz-se uma análise qualitativa das propriedades de convergência de uma extensão do modelo básico desenvolvido na seção anterior. Em tal extensão é levado em consideração um "efeito congestionamento" que pode ser gerado pela migração, devido à existência de um fator de produção fixo na economia, que leva ao aparecimento de uma espécie de retornos decrescentes de escala. Nas considerações finais são resumidos os principais resultados do artigo. 


\section{MACRODINAMMICA DE CRESCIMENTO SOLOW-SWAN COM MIGRAÇÃO: O MODELO DE REFERENNCLA}

Como é bem conhecido, o modelo de crescimento Solow-Swan é construído como uma parábola de uma economia fechada que produz um único bem, que pode ser utilizado tanto como bem de consumo quanto como bem de capital. Em tal cenário é suposto que a poupança $S$ é uma fração constante e exogenamente determinada $0<s<1$ da renda $Y$, que toda a poupança é convertida automaticamente em investimento, $I=S$, e que a população, suposta igual à força de trabalho, cresce a uma taxa exógena constante $n>0$. As possibilidades tecnológicas da economia são sintetizadas em uma função de produção neoclássica bem comportada homogênea de grau um com progresso técnico exógeno do tipo Harrod-neutro: ${ }^{3}$

$$
Y=F(K, \hat{L}), \operatorname{com} \frac{\partial F}{\partial K}>0, \frac{\partial F}{\partial \hat{L}}>0, \frac{\partial^{2} F}{\partial K^{2}}<0 \text { e } \frac{\partial^{2} F}{\partial \hat{L}^{2}}<0
$$

para todo $K>0$ e $\hat{L}>0$, sendo $\hat{L} \equiv L e^{x t}$ a quantidade de trabalho em unidades de eficiência $^{4}$ e $x>0$ a taxa de progresso técnico aumentador de trabalho suposta constante e exogenamente determinada. Além disso, supõe-se que o estoque de capital apresenta uma taxa de depreciação exógena e constante $0<\delta<1$.

A partir de tais premissas pode-se deduzir a conhecida equação fundamental de crescimento Solow-Swan com progresso técnico Harrod-neutro:

$$
\dot{k}=s f(k)-\eta k
$$

sendo $k \equiv K / \hat{L}$ a razão capital-trabalho efetivo, $f(k) \equiv F(k, 1)$ a função de produção na forma intensiva em unidades de eficiência e $\eta \equiv \delta+n+x$ a taxa de depreciação efetiva.

Barro e Sala-i-Martin (2001, cap. 9, sec. 9.1) incorporam a migração nesta versão padrão do modelo Solow-Swan. No restante desta seção apresenta-se tal extensão, que será utilizada no presente artigo como um modelo de referência. Supondo que as populaçóes da economia doméstica $L$ e do resto do mundo crescem à mesma taxa cons-

3 Será usada aqui basicamente a mesma notação de Barro e Sala-i-Martin (2001). Uma exceção importante diz respeito à razão capital-trabalho em unidades de eficiência, a qual estes autores associam o símbolo $\hat{k}$, e aqui, por conveniência, será utilizado simplesmente $k$.

4 Daqui em diante, onde for conveniente será utilizado o termo "efetivo" como sinônimo de "unidades de eficiência". 
tante $n>0$, a taxa de variação da população doméstica pode ser expressa como segue:

$$
\dot{L}=n L+M
$$

na qual $M$ é a migração líquida por unidade de tempo. Quando $M>0$, a taxa de variação da força de trabalho doméstica supera a do resto do mundo, ou seja, a economia doméstica é uma receptora líquida de mão-de-obra. Quando $M<0$, o oposto ocorre, de maneira que a economia torna-se uma fornecedora líquida de mão-de-obra.

Geralmente os migrantes não carregam consigo grande quantidade de capital físico, mas levam uma quantidade considerável de capital humano. Tal como em Barro e Sala-i-Martin (2001, p. 286-287), trabalhar-se-á com um conceito amplo de capital que incorpore estes dois tipos. Seja $\tilde{\kappa}$ a quantidade de capital que cada migrante carrega consigo. A variação no estoque de capital agregado da economia doméstica passa, portanto, a depender também do saldo migratório, isto é:

$$
\dot{K}=s F(K, \hat{L})-\delta K+\tilde{\kappa} M
$$

Utilizando (1.1), (1.3) e (1.4) a equação fundamental de crescimento Solow-Swan com migração pode ser escrita como segue:

$$
\dot{k}=s f(k)-\eta k-(k-\kappa) m
$$

na qual $\kappa$ é a quantidade de capital em unidades de eficiência que cada migrante carrega consigo e $m \equiv M / L$ o fluxo migratório líquido em unidades de população doméstica. Seguindo Barro e Sala-i-Martin (2001, p. 288), assumir-se-á que o resto do mundo encontra-se próximo ao estado estacionário, de maneira que $\kappa$ pode ser considerado constante.

Estes autores argumentam que o fluxo migratório líquido mantém uma relação positiva com o diferencial de salários reais efetivos entre a economia doméstica e o resto do mundo e postulam a seguinte função migração (Barro e Sala-i-Martin, 2001, p. 288):

$$
m=m(k), \text { com } m^{\prime}(k)>0 \text { para todo } k>0
$$

Suponha-se constante o salário do resto do mundo em um nível $\omega>0$. Uma elevação da razão capital-trabalho efetivo da economia doméstica aumenta o seu salário real 
efetivo. Isto melhora, por sua vez, o diferencial de salários efetivos em favor da economia doméstica e, conseqüentemente, aumenta o saldo migratório líquido desta economia. Seja $\tilde{k}$ o valor da razão capital-trabalho efetivo que torna nulo o saldo migratório líquido, ou seja, tal que $m(\tilde{k})=0 .{ }^{5}$ Para valores $k<\tilde{k}(k>\tilde{k})$, portanto, a economia é uma fornecedora (receptora) líquida de mão-de-obra.

Considerando a função migração (1.6) e a equação fundamental de crescimento Solow-Swan com migração (1.5), a equação diferencial que passa a governar a transição de estado da economia doméstica é:

$$
\dot{k}=s f(k)-\eta k-(k-\kappa) m(k)
$$

A existência e unicidade da trajetória de crescimento equilibrado podem ser demonstradas, como fizeram Barro e Sala-i-Martin (2001, p. 289-290), com base em um diagrama de crescimento reproduzido na Figura 1.

\section{FIGURA 1 - DIAGRAMA DE CRESCIMENTO DO MODELO DE REFERENCIA}

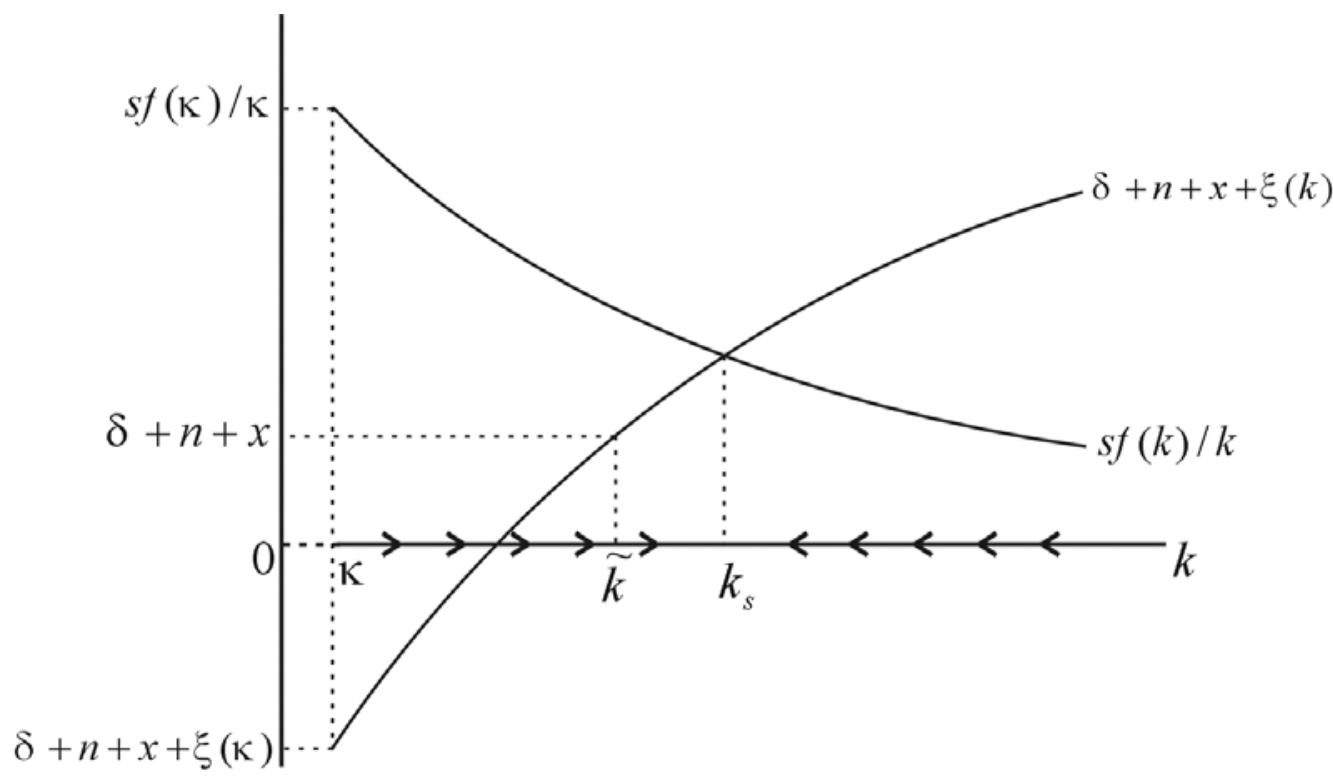

Fonte: Barro e Sala-i-Martin (2001, p. 290). Adaptada pelo autor.

5 A existência de tal valor é suposta por Barro e Sala-i-Martin (2001, p. 288). Se $\tilde{k}$ existe, ele é único, pois $m^{\prime}(k)>0$ para todo $k>0$. 
Deste diagrama pode-se obter informações sobre o comportamento da taxa de crescimento da razão capital-trabalho efetivo, determinada a partir de (1.7):

$$
\frac{\dot{k}}{k}=s \frac{f(k)}{k}-\eta-\xi(k)
$$

na qual $\xi(k) \equiv[1-(\kappa / k)] m(k)$.

A partir da hipótese sobre tecnologia (1.1) segue que o produto médio do capital $f(k) / k$ é uma função estritamente decrescente da razão capital-trabalho efetivo. ${ }^{6}$ Considerando as condições de $\operatorname{Inada}^{7}$ fica garantido que:

$$
\lim _{k \rightarrow 0^{+}} s f(k) / k=+\infty \text { e } \lim _{k \rightarrow+\infty} s f(k) / k=0
$$

A taxa de depreciação efetiva, ${ }^{8} \eta+\xi(k)$, é uma função estritamente crescente da razão capital-trabalho efetivo, pois Barro e Sala-i-Martin (2001, p. 289) supõem que $k>\kappa \mathrm{e}$

$$
\xi^{\prime}(k)=\frac{\kappa}{k^{2}} m(k)+\left(1-\frac{\kappa}{k}\right) m^{\prime}(k)>0 \text { para qualquer } m(k)
$$

Desde que $\tilde{k}>\kappa$ e $\tilde{k}<\infty$, segue que:

$$
\lim _{k \rightarrow \kappa^{+}}[1-(\kappa / k)] m(k)<0 \text { e } \lim _{k \rightarrow \infty}[1-(\kappa / k)] m(k)>0
$$

A partir dos limites (1.8) e (1.10) e da continuidade de (1.7a), infere-se, pelo teorema do valor intermediário, que existe um valor $k_{s}>\kappa$, tal que a taxa de crescimento da razão capital-trabalho efetivo é nula. Dado que a produtividade média do capital é uma função estritamente decrescente da razão capital-trabalho efetivo, a unicidade de $k_{s}$ é decorrência direta da suposição (1.9). ${ }^{9}$

$6 \quad \frac{d}{d k} \frac{f(k)}{k}=\frac{-\left[f(k)-k f^{\prime}(k)\right]}{k^{2}}<0$, pois $\frac{\partial F}{\partial \hat{L}}=f(k)-k f^{\prime}(k)>0$ por hipótese.

7 Que são $\lim _{K \rightarrow 0^{+}} \frac{\partial F}{\partial K}=\lim _{\hat{L} \rightarrow 0^{+}} \frac{\partial F}{\partial \hat{L}}=\infty$ e $\lim _{K \rightarrow \infty} \frac{\partial F}{\partial K}=\lim _{\hat{L} \rightarrow \infty} \frac{\partial F}{\partial \hat{L}}=0$. Estas condições implicam $\lim _{k \rightarrow 0^{+}} f^{\prime}(k)=++\infty$ e $\lim _{k \rightarrow \infty} f^{\prime}(k)=0$ (cf. Barro e Sala-i-Martin, 2001, p. 16-17).

8 Na qual já se encontra incorporado o efeito da migração sobre o estoque de capital doméstico.

9 Pois, supondo (1.9), fica garantido que $\frac{d}{d k} \frac{\dot{k}}{k}=\frac{-s\left[f(k)-k f^{\prime}(k)\right]}{k^{2}}-\xi^{\prime}(k)<0$ para todo $k>\kappa$. 
Nota-se que, a priori, existem três possíveis configurações do estado estacionário. Caso $k_{s}<\tilde{k}$, ter-se-á $m\left(k_{s}\right)<0$ e, portanto, a economia doméstica será perpetuamente uma fornecedora líquida de mão-de-obra. Se $k_{s}>\tilde{k}$ (cf. Figura 1), ter-se-á $m\left(k_{s}\right)>0$ e, portanto, a economia doméstica será uma receptora líquida de mão-de-obra em seu estado estacionário. Finalmente, caso $k_{s}=\tilde{k}$, a economia doméstica apresentará um saldo migratório nulo e sua população crescerá à mesma taxa que o resto do mundo. É importante frisar que neste modelo não se determina a distribuição da população mundial entre a economia doméstica e o resto do mundo.

A estabilidade assintótica da trajetória de crescimento equilibrado pode ser demonstrada utilizando-se novamente o diagrama de crescimento da Figura 1. Se $k<k_{s}\left(k>k_{s}\right)$, a produtividade média do capital supera a (é superada pela) taxa de depreciação efetiva e, portanto, a razão capital-trabalho efetivo cresce (decresce). Enfim, se a razão capital-trabalho efetivo inicial difere da razão capital-trabalho efetivo do estado estacionário, a economia converge assintoticamente para esta última.

Como visto, o modelo exposto nesta seção trata a determinação dos fluxos migratórios com base em uma função migração postulada. Há várias formas de prover microfundamentos para tal função. O presente trabalho propõe derivá-la como uma dinâmica de seleção, deduzida a partir da hipótese de que quanto mais baixo é o salário efetivo numa economia, maior a proporção de indivíduos insatisfeitos que migram para outras economias em busca de maiores salários. Cabe salientar que a função migração a ser derivada na próxima seção não terá como argumento apenas a razão capital-trabalho efetivo da economia doméstica, mas também a fração da população mundial que pertence à economia doméstica. Isto implica a consideração explícita da influência da distribuição da população mundial sobre o processo de acumulação de capital da economia doméstica.

\section{A MIGRAÇÃO ENTRE A ECONOMIA DOMÉSTICA E O RESTO DO MUNDO COMO UMA DINAMMICA SATISFICING}

Nesta seção deriva-se um modelo de jogo evolucionário que determina o fluxo migratório líquido da economia doméstica a cada período supondo livre mobilidade de trabalho. Dividir-se-á, portanto, a população mundial em duas partes: uma delas a proporção $\ell$ da população mundial que trabalha na economia doméstica e a outra a proporção restante, $1-\ell$, que trabalha no resto do mundo. Embora possa haver heterogeneidade de salários efetivos entre a economia doméstica e o resto do mundo, supõe- 
se que há uniformidade de salários efetivos na economia doméstica, bem como no resto do mundo.

Considera-se que um trabalhador $i$ ao receber seu salário efetivo $f(k)-k f^{\prime}(k)$, se for da economia doméstica, ou $\omega$, se for do resto do mundo, o compara com um nível alvo $\bar{w}_{i}$ que o satisfaria, ${ }^{10}$ daqui em diante sinteticamente denominado meta salarial. Se o salário efetivo ganho pelo trabalhador $i$ for igual ou superior a sua meta salarial, ele não cogitaria migrar. Entretanto, caso a meta salarial não seja alcançada, o trabalhador doméstico torna-se um migrante em potencial. ${ }^{11}$

A meta salarial de um indivíduo qualquer depende, entre outras coisas, de características idiossincráticas como, por exemplo, o grau com que deseja melhorar seu padrão de vida ou ascender socialmente. Assumir-se-á que tal meta é determinada aleatoriamente de maneira independente entre os indivíduos e no tempo. Mais precisamente, supõe-se que a meta salarial $\bar{w}_{i}$ é uma variável aleatória com função de distribuição acumulada $G: \mathfrak{R}_{++} \rightarrow[0,1]$ continuamente diferenciável. Assim, a probabilidade de selecionar aleatoriamente um indivíduo $i$ cuja meta salarial $\bar{w}_{i}$ seja menor ou igual ao salário efetivo vigente nesta economia, $w_{i}$ é dada por $\operatorname{Pr}\left(\bar{w}_{i} \leq w_{i}\right)=G\left(w_{i}\right)$ e, portanto, a probabilidade de um indivíduo $i$, tomado aleatoriamente, receber um salário abaixo de sua meta salarial é simplesmente:

$$
\operatorname{Pr}\left(w_{i}<\bar{w}_{i}\right)=1-G\left(w_{i}\right)
$$

Em outras palavras, com esta probabilidade encontra-se aleatoriamente um indivíduo $i$ não satisfeito com seu salário e que está revisando sua escolha relativa ao país onde trabalhar.

A probabilidade de um emigrante de qualquer parte do resto do mundo escolher a economia doméstica em análise é igual à freqüência com que esta estratégia é adotada pela população mundial, dada pela fração da população mundial trabalhando na economia doméstica $\ell$. Logo, considerando (2.1), a imigração estimada para a economia doméstica em unidades de população mundial é:

$$
[1-G(\omega)] \ell(1-\ell)
$$

10 Em Vega-Redondo (1996, p. 91) o nível de referência do pay-off é denominado target level of satisfaction.

11 A derivação de uma satisficing dynamics associada ao fluxo migratório exposta adiante segue as mesmas linhas da derivação de uma satisficing dynamics geral encontrada em Vega-Redondo (1996, p. 91). 
Desde que a economia mundial está dividida em duas regióes, a probabilidade do emigrante da economia doméstica escolher qualquer outra economia para trabalhar coincide com a fração da população mundial que está trabalhando no resto do mundo $(1-\ell)$. Portanto, dada a probabilidade $(2.1)$, a emigração da economia doméstica em unidades de população mundial é estimada em:

$$
\left[1-G\left(f(k)-k f^{\prime}(k)\right)\right](1-\ell) \ell
$$

O saldo migratório líquido da economia doméstica, $\dot{\ell}$, estabelecido como a diferença entre o influxo (2.2) e o efluxo (2.3), é expresso como: ${ }^{12}$

$$
\dot{\ell}=\ell(1-\ell) \phi(k)
$$

em que $\phi(k) \equiv G\left(f(k)-k f^{\prime}(k)\right)-G(\omega)$. Essa equação diferencial é uma dinâmica de seleção ou dinâmica evolucionária, ${ }^{13}$ que apresenta a propriedade de monotonicidade nos pay-offs ${ }^{14}$ se $G^{\prime}\left(w_{i}\right)>0$. Ou seja, a proporção com que a estratégia "trabalhar na economia doméstica" é adotada aumenta (diminui) se, e somente se, o payoff desta estratégia (o salário efetivo da economia doméstica) supera o (é superado pelo) pay-off da estratégia "trabalhar no resto do mundo" (o salário efetivo do resto do mundo).

Deduzida a dinâmica de seleção associada ao processo de migração, cabe estabelecer explicitamente a relação entre tal dinâmica e a função migração postulada em (1.6). Para um dado instante inicial $t_{0}$, a população mundial encontra-se em um nível $N_{0}>$ 0 . Como a população mundial cresce a uma taxa constante e exógena $n>0$, a população mundial em um dado instante $t \geq t_{0}$ será:

$$
N=N_{0} e^{n\left(t-t_{0}\right)}
$$

Lembrando que $L$ denota a população da economia doméstica no instante $t$, a fração da população mundial trabalhando na economia doméstica ou, equivalentemente, a população doméstica em unidades de população mundial no instante $t$ é:

12 Seria possível apresentar uma derivação alternativa da dinâmica de seleção (2.4) baseada em custos de mudança aleatórios, que incluiria não só custos monetários explícitos mas também custos psicológicos gerados pela migração. Uma derivação nesta linha pode ser encontrada em Silveira e Sanson (2004, Apêndice 1).

13 Sobre tal dinâmica consulte Ponti (2002), Vega-Redondo (1996, cap. 4) e Weibull (1995, cap. 4).

14 Tal propriedade desempenha um papel na modelagem de processos evolucionários em ambientes sociais análogo ao mecanismo de seleção natural em ambientes biológicos. 


$$
\ell=\frac{L}{N_{0} e^{n\left(t-t_{0}\right)}}
$$

A partir de (2.6) infere-se que:

$$
\frac{\dot{\ell}}{\ell}=\frac{\dot{L}}{L}-n
$$

então, considerando (1.3) e a definição $m \equiv M / L$, conclui-se que a taxa de crescimento da fração da população mundial residindo na economia doméstica é igual ao fluxo migratório líquido em unidades de população doméstica, ou seja:

$$
\frac{\dot{\ell}}{\ell}=m
$$

Considerando a dinâmica de seleção (2.4) e a relação (2.8), fica demonstrado que a função migração (1.6) está sendo substituída por:

$$
m=(1-\ell) \phi(k)
$$

Essa função migração é uma expressão do processo migratório com fundamentos microeconômicos oriundos de diferenças idiossincráticas entre as metas salariais dos trabalhadores. Tal como na função migração postulada em (1.6), na função migração (2.4a) um aumento da razão capital-trabalho efetivo doméstica, ao elevar o salário real doméstico, aumenta o fluxo migratório líquido para uma dada distribuição da população mundial. Além disso, observa-se que o nível do saldo migratório líquido depende do tamanho da população do resto do mundo e do diferencial de salários efetivos entre a economia doméstica e o resto do mundo.

O fluxo migratório líquido será nulo caso toda a população mundial seja completamente absorvida pela economia doméstica $(\ell=1)$ ou a razão capital-trabalho efetivo atinja o nível $k_{c}$, definido implicitamente por:

$$
f\left(k_{c}\right)-k_{c} f^{\prime}\left(k_{c}\right)=\omega
$$

no qual o salário efetivo doméstico iguala-se ao salário efetivo do resto do mundo e, conseqüentemente, $\phi\left(k_{c}\right)=G\left(f\left(k_{c}\right)-k_{c} f^{\prime}\left(k_{c}\right)\right)-G(\omega)=0$. Como será demonstrado nas duas próximas seções, o valor $k_{c}$ exerce no modelo evolucionário aqui proposto o mesmo papel que o desempenhado pelo valor $\tilde{k}$ no modelo de referência apresentado 
na seção anterior. Tal como Barro e Sala-i-Martin (2001, p. 288) fizeram com respeito ao valor $\tilde{k}$, será assumido que o valor $k_{c}$ existe. Sua unicidade, considerando (2.9), decorre da premissa de produtividade marginal decrescente do trabalho.

Finalmente, cabe salientar que Barro e Sala-i-Martin (2001, p. 288, nota 4) adotam a seguinte premissa com respeito à função migração (1.6): "assumimos que imigração e emigração não ocorrem simultaneamente, tal que migração líquida e bruta coincidem. Geralmente, a beterogeneidade do capital humano ou de outras variáveis poderia fazer com que fluxos brutos excedessem fluxos líquidos." (Tradução e grifos meus). Na função migração (2.4a), entretanto, há imigração e emigração simultaneamente na economia doméstica devido à heterogeneidade dos indivíduos com relação à meta salarial. Assim, a equalização entre os salários efetivos da economia doméstica e do resto do mundo, que torna o saldo migratório líquido nulo, significa simplesmente que influxo e efluxo de migrantes na economia doméstica se contrabalançam. Ou seja, mesmo com a igualação dos salários efetivos entre a economia doméstica e o resto do mundo há indivíduos nestas regiões que estão insatisfeitos com seus salários e migram em busca de melhores remunerações.

De posse da dinâmica de seleção (2.4) e da nova função migração (2.4a), passa-se à análise das implicações da interação entre o processo migratório e a dinâmica de crescimento Solow-Swan.

\section{A INTERAÇÃO ENTRE A DINÂMICA MIGRATÓRIA SATISFICING E A DINAMICA DE CRESCIMENTO SOLOW-SWAN}

Introduzindo (2.4a) na equação fundamental de crescimento com migração (1.7) resulta uma equação de crescimento que juntamente com a dinâmica de seleção (2.4) passam a determinar a trajetória de crescimento da economia doméstica:

$$
\begin{aligned}
& \dot{k}=s f(k)-\eta k-(k-\kappa)(1-\ell) \phi(k) \\
& \dot{\ell}=\ell(1-\ell) \phi(k)
\end{aligned}
$$

O espaço de estado deste sistema é o conjunto $\Theta=\left\{(k, \ell) \in \mathfrak{R}_{+}^{2}: 0<\ell \leq 1\right.$ e $\left.k>\kappa>0\right\}$.

Se no estado estacionário houver um diferencial de salários efetivos entre a economia doméstica e o resto do mundo não nulo, ocorre um tipo extremo de distribuição da população mundial. Nesse último a população do resto do mundo é completamente absorvida pela economia doméstica $(\ell=1)$ e a razão capital-trabalho efetivo é igual 
à razão capital-trabalho efetivo estacionária Solow-Swan $k_{s}$, definida implicitamente pela conhecida condição:

$$
s f\left(k_{s}\right)-\eta k_{s}=0
$$

que torna a razão capital-trabalho efetivo doméstica estacionária. ${ }^{15}$ Do ponto de vista da teoria dos jogos evolucionários, esse estado estacionário pode ser caracterizado como um equilíbrio de estratégia pura, pois nele todos os indivíduos adotam a estratégia "trabalhar na economia doméstica".

Se no estado estacionário os salários reais efetivos da economia doméstica e do resto do mundo são iguais, há um saldo migratório nulo e um único valor da razão capitaltrabalho efetivo $k_{c}$, definido implicitamente por (2.9), tal que a economia doméstica iguala o investimento per capita e a depreciação líquida, independentemente da distribuição da população mundial. Em outras palavras, há um continumm de pontos de equilíbrio $\left\{(k, \ell) \in \Theta \subset \mathfrak{R}_{+}^{2}: k=k_{s}=k_{c}\right\}$ se no estado estacionário os salários reais efetivos da economia doméstica e do resto do mundo forem iguais. Com exceção do ponto $\left(k_{c}, 1\right)$, os demais pontos do continumm $\left\{(k, \ell) \in \Theta \subset \mathfrak{R}_{+}^{2}: k=k_{s}=k_{c}\right\}$ são, do ponto de vista da teoria dos jogos evolucionários, equilíbrios de estratégia mista, já que existem tanto indivíduos que adotam a estratégia "trabalhar na economia doméstica" quanto indivíduos que optam pela estratégia "trabalhar no resto do mundo".

Os resultados sobre a existência de equilíbrios no sistema (3.1) expostos nos dois últimos parágrafos são sintetizados na proposição adiante e demonstrados formalmente no Apêndice 1.

ProposiÇÃo 1. Sejam $k_{c}$ e $k_{s}$ valores definidos implicitamente por (2.9) e (3.2), respectivamente. Se $k_{s} \neq k_{c}$, então o sistema (3.1) apresenta, supondo $s f(\kappa)-\eta \kappa>0$, um único estado de equilíbrio isolado $\left(k_{s}, 1\right) \in \Theta$. Se $k_{s}=k_{c}$, então existe um continumm de equilíbrios, ou seja, todos os pontos pertencentes ao subconjunto $\left\{(\ell, k) \in \Theta \subset \mathfrak{R}_{+}^{2}: k=k_{s}=k_{c}\right\}$ são estados de equilíbrio do sistema (3.1).

15 Este estado estacionário é parametrizado pelo mesmo conjunto de variáveis exógenas que o estado estacionário do modelo Solow-Swan de uma economia fechada com progresso técnico Harrod-neutro. Além disso, os efeitos de tais variáveis exógenas sobre o estado estacionário são idênticos nos dois casos. 
Prova: Apêndice 1.

As características qualitativas da dinâmica gerada pelo sistema (3.1) encontram-se sintetizadas nos diagramas de fase na Figura 2. Considerando a dinâmica de seleção (2.4), o fluxo migratório líquido é nulo em qualquer estado no qual a fração da população mundial trabalhando na economia doméstica seja igual a um. Além disso, qualquer estado caracterizado por uma razão capital-trabalho efetivo igual a $k_{c}$ gera também um fluxo migratório líquido nulo, pois o salário efetivo doméstico se iguala ao salário efetivo do resto do mundo. Portanto, no locus na forma de um "T" definido pelo conjunto $\{(k, \ell) \in \Theta: \ell=1\} \bigcup\left\{(k, \ell) \in \Theta: k=k_{c}\right\}$ tem-se $\dot{\ell}=0$. Para estados nos quais $k>k_{c}$, o salário real efetivo doméstico é superior ao salário real efetivo do resto do mundo havendo, conseqüentemente, um saldo migratório líquido para a economia doméstica, ou seja, $\dot{\ell}>0$. Se $k<k_{c}$, o oposto acontece, isto é, $\dot{\ell}<0$.

FIGURA 2 - MACRODINÂMICAS DE CRESCIMENTO

(a) Caso $k_{s}>k_{c}$

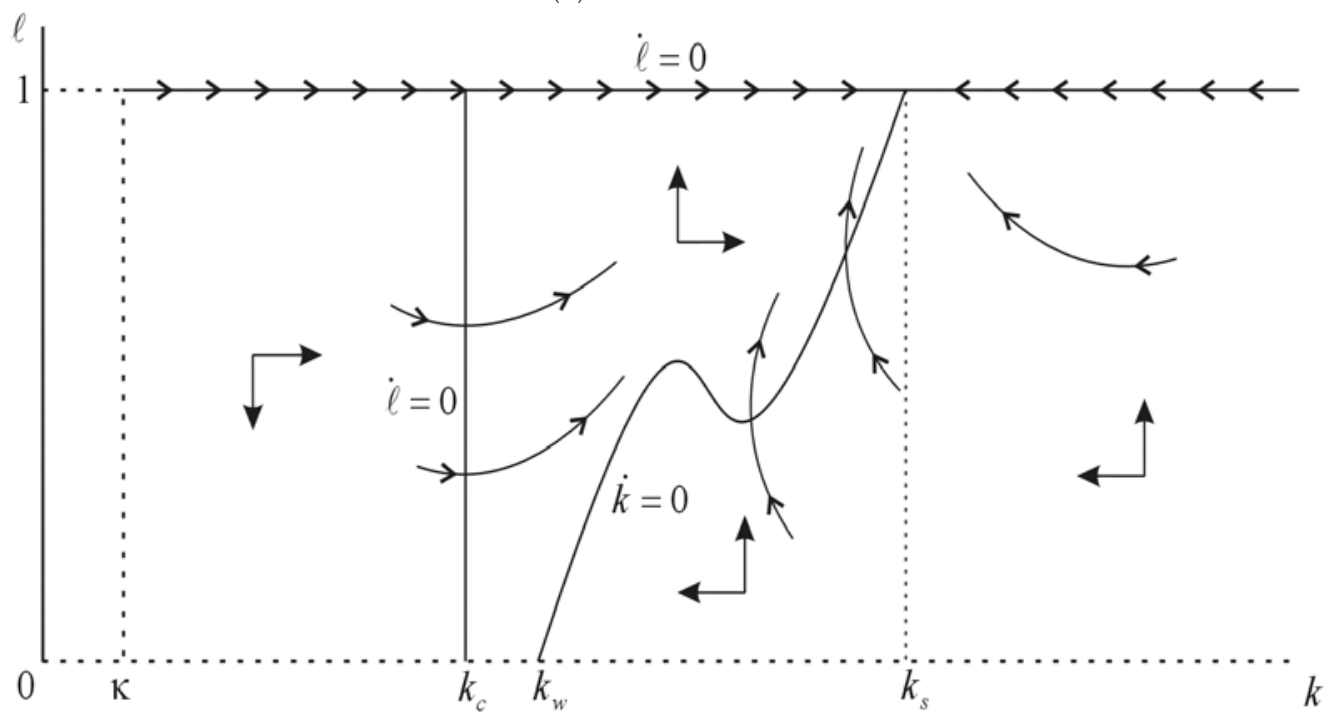


(b) Caso $k_{s}<k_{c}$

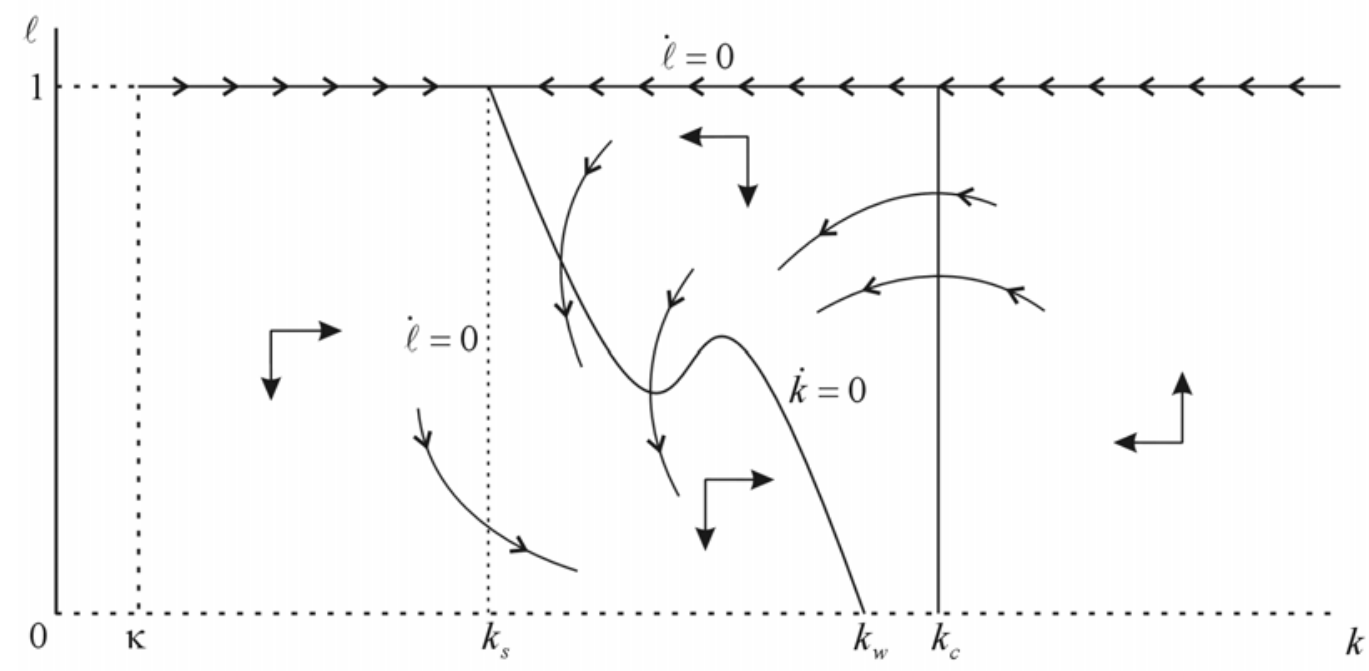

(c) Caso $k_{s}=k_{c}$

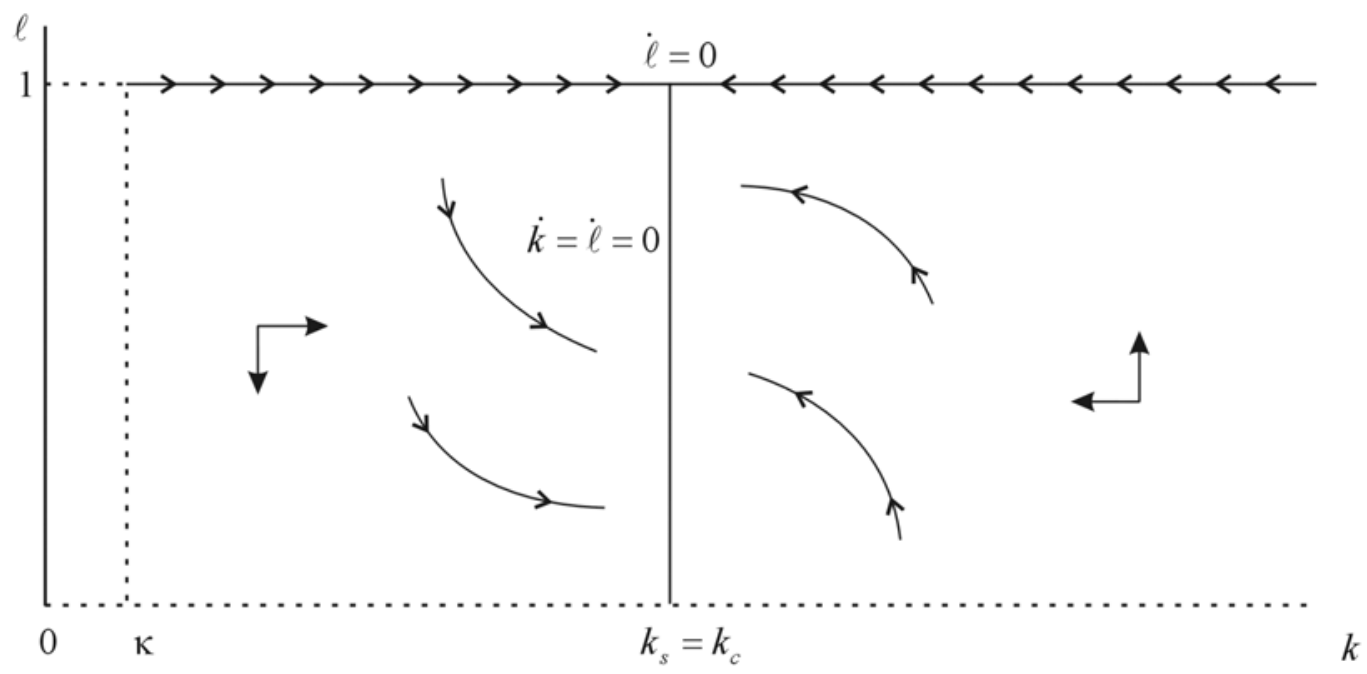

A partir da primeira equação em (3.1) obtém-se a expressão que define a isoclina $\dot{k}=0$, daqui em diante denominada curva de demarcação $k$ :

$$
\ell=1-\frac{s f(k)-\eta k}{(k-\kappa) \phi(k)} \equiv \psi k
$$

definida em todo $k \in \Theta$ tal que $(k-\kappa) \phi(k) \neq 0$. Como, por hipótese, $k>\kappa$, a curva de demarcação $k$ é definida para qualquer $k \neq k_{c}$. 
Para grafar a curva de demarcação $k$ deve-se estabelecer previamente as posições relativas de $k_{c}$ e $k_{s}$ no eixo $k$ do plano de fases. Suponha que no estado estacionário SolowSwan a economia doméstica seja uma receptora líquida de mão-de-obra, ou seja, $k_{s}>k_{c}$ (cf. Figura 2a). Considerando (3.3), para $k=k_{s}>k_{c}$ tem-se $\psi\left(k_{s}\right)=0$ e, portanto, $\ell=1$. A curva de demarcação $k$, como deveria ser, cruza a curva de demarcação $\ell$ (isoclina $\ell=0$ ) no estado estacionário com estratégia pura $\left(k_{s}, 1\right)$.

Supondo $0<\ell \leq 1$, infere-se que a parte economicamente relevante da curva de demarcação $k$ encontra-se na região $\left\{(k, \ell) \in \Theta: k_{c}<k \leq k_{s}\right\}$. Observando a primeira equação diferencial do sistema (3.1), vê-se que para qualquer $k \in\left(\kappa, k_{c}\right]$ o investimento per capita supera a taxa de depreciação líquida, e como o salário efetivo doméstico é menor ou igual ao salário efetivo do resto do mundo, o saldo migratório líquido negativo reforça este efeito do investimento, de modo que a razão capital-trabalho efetivo cresce, isto é, $\dot{k}>0$. Exatamente o oposto acontece se $k \in\left(k_{s}, \infty\right)$, ou seja, $\dot{k}<0$. Enfim, o subespaço de estados $\left\{(k, \ell) \in \Theta: k_{c}<k \leq k_{s}\right\}$ é onde a economia doméstica pode apresentar uma razão capital-trabalho efetivo estacionária $(\dot{k}=0)$.

A inclinação da curva de demarcação $k$ no subespaço $\left\{(k, \ell) \in \Theta: k_{c}<k \leq k_{s}\right\}$ é obtida derivando a função (3.3) com relação à razão capital-trabalho efetivo:

$$
\psi^{\prime}(k)=\frac{[s f(k)-\eta k]\left[\phi(k)+(k-\kappa) \phi^{\prime}(k)\right]-\left[s f^{\prime}(k)-\eta\right](k-\kappa) \phi(k)}{[(k-\kappa) \phi(k)]^{2}}
$$

No intervalo $\left(k_{c}, k_{s}\right)$ tem-se $s f(k)-\eta k>0, \quad \phi(k)>0, \quad k-\kappa>0 \quad$ e $\phi^{\prime}(k)=-G^{\prime}(\cdot) k f^{\prime \prime}(k)>0$, porém a expressão $s f^{\prime}(k)-\eta$ apresenta sinal indeterminado. Logo, a função (3.4) pode não ser monótona no intervalo $\left(k_{c}, k_{s}\right)$.

A função (3.4) apresenta o seguinte comportamento na fronteira do intervalo $\left(k_{c}, k_{s}\right]$ :

$$
\begin{aligned}
& \lim _{k \rightarrow k_{c}^{+}} \psi(k)=1-\left[\frac{s f\left(k_{c}\right)-\eta k_{c}}{k_{c}-\kappa}\right] \lim _{k \rightarrow k_{c}^{+}} \frac{1}{\phi(k)}=1-\left[\frac{s f\left(k_{c}\right)-\eta k_{c}}{k_{c}-\kappa}\right] . \infty=-\infty \text { e } \\
& \lim _{k \rightarrow k_{s}^{-}} \psi(k)=1-\frac{\lim _{k \rightarrow k_{s}^{-}} s f(k)-\eta k}{\left(k_{s}-\kappa\right) \kappa\left(k_{s}\right)}=1-\frac{0}{\left(k_{s}-\kappa\right) \phi\left(k_{s}\right)}=1
\end{aligned}
$$

Então, pelo teorema do valor intermediário, infere-se que existe um $k_{w}$, tal que $\psi\left(k_{w}\right)=0$. Suponha que $k_{w}$ é único. Como 


$$
\psi\left(k_{s}\right)=\frac{\eta-s f^{\prime}\left(k_{s}\right)}{\left(k_{s}-\kappa\right) \phi\left(k_{s}\right)}>0
$$

deve-se ter $\psi^{\prime}(k)>0$ em uma vizinhança em torno de $k_{w}$, pois do contrário $k_{w}$ não seria único. Dessa forma, considerando (3.4), conclui-se que em uma vizinhança à direita de $k_{w}$ a curva de demarcação $k$ é positivamente inclinada. Considerando (3.6), em uma vizinhança suficientemente pequena de $k_{s}$ a curva de demarcação $k$ é também positivamente inclinada. Portanto, a curva de demarcação $k$ pode apresentar uma reversão de inclinação somente no interior do intervalo $\left(k_{w}, k_{s}\right]$, como ilustrado na Figura 2a. Se isso ocorre ou não, dada a unicidade do ponto $\left(k_{w}, 0\right)$, é irrelevante para as conclusões qualitativas sobre a dinâmica do sistema que seguem.

Para se ter $0<\ell \leq 1$, a função $\psi$ deve obedecer as restrições $0<\psi(k) \leq 1$. Desde que $s f(k)-\eta k \geq 0, k-\kappa>0$ e $\phi(k)>0$ no intervalo $\left(k_{c}, k_{s}\right]$, a restrição anterior é satisfeita se para todo $k \in\left(k_{c}, k_{s}\right]$ :

$$
s f(k)-\eta k<(k-\kappa) \phi(k)
$$

condição essa suposta válida daqui para frente.

No intervalo $\left(k_{c}, k_{s}\right)$ tem-se:

$$
\frac{\partial \dot{k}}{\partial \ell}=(k-\kappa) \phi(k)>0
$$

o que indica que para uma dada razão capital-trabalho efetivo aumentos da fração de indivíduos residindo na economia doméstica geram uma expansão da taxa de variação da razão capital-trabalho efetivo. Então, pontos localizados na região $\left\{(k, \ell) \in \Theta: k_{c}<k \leq k_{s}\right\}$ e abaixo (acima) da curva de demarcação $k$, apresentam uma taxa de variação da razão capital-trabalho efetivo negativa (positiva). Isso completa a construção do diagrama de fase da Figura 2a, do qual se conclui que o estado estacionário com estratégia pura $\left(k_{s}, 1\right)$ é um atrator e a economia doméstica tende a absorver toda a população do resto do mundo.

Na hipótese de a economia doméstica ser uma fornecedora líquida de mão-de-obra no estado estacionário Solow-Swan, ou seja, $k_{s}<k_{c}$, uma análise análoga à realizada anteriormente (cf. Apêndice 2) permite concluir que o estado estacionário com estratégia pura $\left(k_{s}, 1\right)$ torna-se um repulsor e a economia doméstica tende à extinção. 
Há ainda uma terceira possibilidade, a saber, a de que a economia doméstica apresente um saldo migratório líquido nulo, ou seja, que $k_{c}=k_{s}$. Neste caso, a curva de demarcação $k$ coincide com a curva de demarcação $\ell$, havendo, como já demonstrado, um continum de equilíbrios que corresponde ao conjunto $\left\{(k, \ell) \in \Theta: k=k_{s}=k_{c}\right\}$. Assim, para qualquer razão capital-trabalho efetivo menor que a razão capital-trabalho efetivo Solow-Swan, a economia doméstica apresentará um saldo migratório líquido negativo $(\dot{\ell}<0)$ e um investimento per capita superior à depreciação líquida $(\dot{k}>0)$. Os respectivos sinais se invertem caso a razão capital-trabalho efetivo da economia doméstica seja maior que a razão capital-trabalho efetivo Solow-Swan (cf. Figura 2c).

Em suma, se no estado estacionário Solow-Swan a economia doméstica é uma receptora líquida de força de trabalho, tal economia absorverá toda a população mundial. Caso a economia doméstica seja uma fonte líquida de força de trabalho no estado estacionário Solow-Swan, então ela tende à extinção. Finalmente, se a economia doméstica apresentar um saldo migratório líquido nulo no estado estacionário SolowSwan a distribuição da população mundial no estado estacionário dependerá do estado inicial da economia doméstica. A partir da análise qualitativa realizada anteriormente pode-se estabelecer a seguinte proposição concernente ao comportamento dinâmico do modelo:

Proposição 2. Se no estado estacionário com estratégia pura $\left(k_{s}, 1\right) \in \Theta$ tem-se $\left.f\left(k_{s}\right)-k_{s} f^{\prime}\left(k_{s}\right)\right]>\omega$, então $\left(k_{s}, 1\right) \in \Theta$ é um atrator global. Se $\left.f\left(k_{s}\right)-k_{s} f^{\prime}\left(k_{s}\right)\right]<\omega$, então $\left(k_{s}, 1\right) \in \Theta$ é instável, mais precisamente, um ponto de sela cujo ramo estável é o locus $\left\{(k, \ell) \in \Theta: k \neq k_{\mathrm{s}}\right.$ e $\left.\ell=1\right\}$, e para qualquer condição inicial $\left(k_{0}, \ell_{0}\right) \in$ int $\Theta$, tem-se $\ell \rightarrow 0^{+}$. Se $f\left(k_{s}\right)-k_{s} f^{\prime}\left(k_{s}\right)=\omega$, então a economia doméstica converge para um dos estados estacionários com estratégia mista do subespaço $\left\{(k, \ell) \in \Theta: \ell<1\right.$ e $\left.k_{c}<k \leq k_{s}\right\}$, a depender da condição inicial $\left(k_{0}, \ell_{0}\right) \in \operatorname{int} \Theta$.

\section{Prova: Apêndice 2.}

Os resultados qualitativos relativos às dinâmicas do modelo enunciado na proposição anterior diferem dos resultados de livro-texto (cf. Barro e Sala-i-Martin, 2001, subseção 9.1.1). Em primeiro lugar, no modelo aqui proposto não pode haver coexistência da economia doméstica com o resto do mundo caso a primeira seja uma receptora ou fornecedora líquida de mão-de-obra, isto é, a economia doméstica não pode ser uma "perpétua" fornecedora ou receptora de mão-de-obra. Em contraste, no modelo apresentado na primeira seção não aparece essa restrição, a economia doméstica pode ser uma fornecedora ou receptora líquida de máo-de-obra ad infinitum. Em segundo lugar, caso a economia doméstica seja representativa do resto do mundo, isto é, apre- 
sente um saldo migratório líquido nulo devido à igualação dos salários reais efetivos, o tamanho relativo da economia doméstica depende das condições iniciais desta economia. Em outros termos, há um fenômeno de path dependence, ou seja, o tamanho relativo da população doméstica no estado estacionário depende da distribuição inicial da população mundial. No modelo de referência não se extraiu qualquer conclusão sobre o tamanho relativo da população doméstica. Em suma, com o modelo construído nesta seção são alcançados os mesmos resultados relativos à estabilidade assintótica do estado estacionário Solow-Swan com migração e, além disso, obtidos resultados adicionais sobre a distribuição relativa da população mundial.

A conclusão de que a economia doméstica pode ser indefinidamente uma fornecedora ou receptora líquida de mão-de-obra, no caso do modelo de referência, e se extinguir ou absorver toda a população mundial, no caso do modelo evolucionário aqui apresentado, não são resultados empiricamente razoáveis. Para resolver este problema de predição, Barro e Sala-i-Martin (2001, subseção 9.1.3), inspirados em Braun, introduzem uma forma de retornos decrescentes de escala na economia doméstica, supondo que um aumento da população congestiona um recurso disponível em quantidade fixa, tais como recursos naturais ou oferta de bens públicos. Com esta nova suposição, os resultados não condizentes com a evolução histórica das economias são eliminados no modelo de referência. Na próxima seção será avaliada se tal hipótese de retornos decrescentes gerados pela migração é também capaz de eliminar as predições irrealistas do modelo evolucionário aqui desenvolvido.

\section{A INTERAÇÃO ENTRE O PROCESSO MIGRATÓRIO E A DINÂMICA DE CRESCIMENTO NA PRESENÇA DE UM EFEITO CONGESTIONAMENTO}

Seguindo Braun (1993 apud Barro e Sala-i-Martin, 2001, p. 300), admite-se que a economia doméstica é dotada de um dado estoque fixo e exogenamente determinado de um fator de produção, como, por exemplo, recursos naturais ou um bem livre qualquer disponível aos residentes em uma quantidade fixa. Além disso, supõe-se que a dotação per capita deste recurso fixo afeta o produtividade dos fatores de produção utilizados pelas firmas da economia doméstica. Em termos formais, considera-se que as firmas domésticas se defrontam com uma função de produção do tipo:

$$
Y=F(K, \hat{L})(\bar{R} / L)^{\lambda}
$$

na qual $\bar{R}>0$ é uma constante exógena, que representa o estoque fixo de um fator de produção, $\lambda$ é uma constante exógena, tal que $0<\lambda<1$, e $F(K, \hat{L})$ a função de produção neoclássica bem comportada especificada em (1.1). Cada firma doméstica 
toma como dada a população da economia ${ }^{16} \mathrm{e}$, portanto, produz sujeita a uma função de produção com retornos constantes de escala. Todavia, a economia como um todo apresenta retornos decrescentes de escala com relação ao estoque de capital e à população, pois o crescimento da população gera um congestionamento na utilização do fator fixo $\bar{R} \cdot{ }^{17}$

A função (4.1) pode ser expressa na forma intensiva em unidades de eficiência como segue:

$$
f(k)(R / \ell)^{\lambda}
$$

na qual $f(k) \equiv F(k, 1)$ e $R \equiv \bar{R} / N$.

Com base nessa nova função de produção (4.2), o sistema (3.1) transforma-se em:

$$
\begin{aligned}
& \dot{k}=s f(k)(R / \ell)^{\lambda}-\eta k-(1-\ell)(k-\kappa) \varphi(k, \ell) \\
& \dot{\ell}=\ell(1-\ell) \varphi(k, \ell)
\end{aligned}
$$

em que $\varphi(k, \ell) \equiv G\left(\left[f(k)-k f^{\prime}(k)\right](R / \ell)^{\lambda}\right)-G(\omega)$. Esse novo sistema ainda tem como espaço de estados $\Theta=\left\{(k, \ell) \in \mathfrak{R}_{+}^{2}: 0<\ell \leq 1\right\}$.

Essa versão estendida do modelo apresenta apenas um estado estacionário com estratégia pura. Em tal estado a população do resto do mundo é completamente absorvida pela economia doméstica $(\ell=1)$ e a razão capital-trabalho efetivo é igual à razão capital-trabalho efetivo estacionária Solow-Swan $\tilde{k}_{s}$, agora definida implicitamente pela nova condição:

$$
s f\left(\tilde{k}_{s}\right) R^{\lambda}-\eta \tilde{k}_{s}=0
$$

que torna a razão capital-trabalho efetivo doméstica estacionária.

O sistema (4.3) apresenta, também, um único estado estacionário com estratégia mista. Neste estado estacionário existem tanto indivíduos optando pela estratégia "trabalhar na economia doméstica" quanto indivíduos adotando a estratégia "trabalhar

16 Ou seja, toma como exógeno o termo $(\bar{R} / L)^{\lambda}$.

17 Faça $H(K, L) \equiv F(K, \hat{L})(\bar{R} / L)^{\lambda}$. Logo, considerando $(1.1), H(\beta K, \beta L)=\beta^{1-\lambda} H(K, L)$ para todo $\beta>0$. Por hipótese, $0<\lambda<1$, então $0<1-\lambda<1$. A função de produção $(4.1)$, portanto, apresenta retornos decrescentes de escala com respeito a $K$ e $L$. 
no resto do mundo". Tal equilíbrio, denotado por $\left(k^{*}, \ell^{*}\right)$, é definido pelo seguinte conjunto de condições: ${ }^{18}$

$$
\begin{aligned}
& \ell^{*}=\left\{\frac{\left[f\left(k^{*}\right)-k^{*} f^{\prime}\left(k^{*}\right)\right] R^{\lambda}}{\omega}\right\}^{1 / \lambda} \\
& s \frac{f\left(k^{*}\right)}{k^{*}}-\eta\left[\frac{f\left(k^{*}\right)-k^{*} f^{\prime}\left(k^{*}\right)}{\omega}\right]=0
\end{aligned}
$$

A condição (4.5), obtida da segunda equação em (4.3), fornece o valor da fração de indivíduos da população mundial que estão adotando a estratégia "trabalhar na economia doméstica” que gera um salário efetivo doméstico igual ao salário efetivo do resto do mundo. A condição (4.6), obtida a partir de primeira equação em (4.3), define implicitamente a razão capital-trabalho efetivo tal que o investimento per capita iguala-se à taxa de depreciação efetiva quando o salário efetivo doméstico é igual ao salário efetivo do resto do mundo.

A proposição adiante sintetiza a argumentação apresentada nos parágrafos anteriores, explicitando formalmente as condições suficientes para existência dos estados estacionários de estratégias pura e mista.

Proposição 3. Seja $k_{c}$ o valor definido implicitamente por (2.9). Supondo $s f(\kappa) R^{\lambda}-\eta \kappa>0$, existe um único equilíbrio de estratégia pura $\left(\tilde{k}_{s}, 1\right) \in \Theta$, sendo $\tilde{k}_{s}$ definido implicitamente por (4.4). Além disso, há um único equilíbrio de estratégia mista $\left(k^{*}, \ell^{*}\right) \in \Theta$ definido pelas condições $(4.5)$ e (4.6) se $\left[f\left(k^{*}\right)-k^{*} f^{\prime}\left(k^{*}\right)\right] R^{\lambda}<\omega$.

\section{Prova: Apêndice 3.}

Dada a condição (4.6), os impactos de variações da propensão a poupar e da taxa de depreciação efetiva sobre a razão capital-trabalho efetivo de equilíbrio são:

$$
\begin{aligned}
& \frac{\partial k^{*}}{\partial s}=\frac{\omega k^{*} f\left(k^{*}\right)}{s \omega\left[f\left(k^{*}\right)-k^{*} f^{\prime}\left(k^{*}\right)\right]-\eta\left(k^{*}\right)^{3} f^{\prime \prime}\left(k^{*}\right)}>0 \mathrm{e} \\
& \frac{\partial k^{*}}{\partial \eta}=\frac{-\left(k^{*}\right)^{2}\left[f\left(k^{*}\right)-k^{*} f^{\prime}\left(k^{*}\right)\right]}{s \omega\left[f\left(k^{*}\right)-k^{*} f^{\prime}\left(k^{*}\right)\right]-\eta\left(k^{*}\right)^{3} f^{\prime \prime}\left(k^{*}\right)}<0
\end{aligned}
$$

18 A derivação destas condições aparece no transcorrer da demonstração desenvolvida no Apêndice 3. 
Além disso, com base em (4.5) tem-se que:

$$
\frac{\partial \ell^{*}}{\partial k^{*}}=-\frac{R k^{*} f^{\prime \prime}\left(k^{*}\right)}{\lambda \omega}\left[\frac{f\left(k^{*}\right)-k^{*} f^{\prime}\left(k^{*}\right)}{\omega}\right]^{(1-\lambda) / \lambda}>0
$$

ou seja, o tamanho relativo da economia doméstica de equilíbrio varia diretamente com a razão capital-trabalho efetivo de equilíbrio. Logo, o efeito de variações da propensão a poupar sobre o estado estacionário trabalha no mesmo sentido que na versão do modelo de Solow-Swan sem migração, ou seja, um(a) aumento (redução) permanente da propensão a poupar aumenta (diminui) a razão capital-trabalho efetivo e, portanto, aumenta (diminui) a renda per capita e o tamanho relativo da economia doméstica. Por sua vez, uma variação da taxa de depreciação efetiva, via variação da taxa de depreciação do estoque de capital e/ou da taxa natural de crescimento da população e/ou da taxa de progresso técnico, gera uma variação no sentido inverso da razão capital-trabalho efetivo e, conseqüentemente, da renda per capita e do tamanho relativo da economia doméstica.

Com respeito ao impacto do salário real efetivo do resto do mundo infere-se de (4.5) e (4.6):

$$
\begin{aligned}
& \frac{\partial \ell^{*}}{\partial \omega}=-\frac{1}{\lambda \omega}\left\{\frac{\left[f\left(k^{*}\right)-k^{*} f^{\prime}\left(k^{*}\right)\right] R^{\lambda}}{\omega}\right\}^{1 / \lambda}<0 \text { е } \\
& \frac{\partial k^{*}}{\partial \omega}=\frac{(1 / \omega)\left(k^{*}\right)^{2} \eta\left[f\left(k^{*}\right)-k^{*} f^{\prime}\left(k^{*}\right)\right]}{s \omega\left[f\left(k^{*}\right)-k^{*} f^{\prime}\left(k^{*}\right)\right]-\eta\left(k^{*}\right)^{3} f^{\prime \prime}\left(k^{*}\right)}>0
\end{aligned}
$$

Portanto, um aumento (diminuição) do salário real efetivo do resto do mundo, ao induzir uma maior (menor) emigração da economia doméstica, acaba diminuindo (aumentando) o tamanho relativo da economia doméstica e elevando (diminuindo) a razão capital-trabalho efetivo e a renda per capita desta última.

Finalmente, é importante notar que a razão capital-trabalho efetivo do equilíbrio de estratégia mista não depende do grau de homogeneidade da função de produção (4.1), determinado pelo parâmetro $\lambda$. Por sua vez, o tamanho relativo da economia doméstica é afetado pela intensidade dos retornos decrescentes de escala gerados pelo efeito congestionamento. A direção deste impacto é indeterminada, como pode ser constatado pela seguinte derivada obtida de (4.5):

$$
\frac{\partial \ell^{*}}{\partial \lambda}=-\frac{1}{\lambda^{2}}\left\{\frac{\left[f\left(k^{*}\right)-k^{*} f^{\prime}\left(k^{*}\right)\right] R^{\lambda}}{\omega}\right\}^{1 / \lambda} \ln \left(\frac{\omega}{f\left(k^{*}\right)-k^{*} f^{\prime}\left(k^{*}\right)}\right)
$$


Em outros termos, o impacto dos retornos decrescentes de escala devido ao fator escasso depende fundamentalmente do tamanho relativo do salário real efetivo do resto do mundo. Se, por exemplo, tal salário for relativamente alto, $\omega>f\left(k^{*}\right)-k^{*} f^{\prime}\left(k^{*}\right)$, então (4.10) apresenta sinal negativo. Logo, um aumento do efeito congestionamento, ou seja, uma redução do grau de homogeneidade da função de produção (4.1), via elevação de $\lambda$, geraria uma redução do tamanho relativo da economia doméstica.

Com relação às propriedades de estabilidade da economia doméstica, percebe-se que esta poderá absorver toda a população mundial caso ela se encontre suficientemente próxima do estado estacionário de estratégia pura e apresente um salário efetivo maior que o do resto do mundo. Em outras palavras, se a economia doméstica for uma receptora líquida de mão-de-obra no estado estacionário de estratégia pura, tal estado é um atrator local do sistema econômico.

Entretanto, caso a economia seja uma fornecedora de mão-de-obra no estado estacionário de estratégia pura, por apresentar um salário efetivo inferior ao salário efetivo do resto do mundo, mesmo que se encontre suficientemente próxima a tal trajetória de crescimento equilibrado com extinção do resto do mundo, ela não convergirá para esta última. Em outros termos, sob a hipótese de que a economia doméstica seja uma fornecedora líquida de emigrantes no estado estacionário de estratégia pura, tal estado torna-se um repulsor local do sistema econômico.

Independentemente da posição da economia doméstica, no que diz respeito ao saldo migratório líquido, ${ }^{19}$ o estado estacionário com estratégia mista será um atrator local do sistema econômico. Ou seja, se a economia doméstica encontrar-se suficientemente próxima da trajetória de crescimento equilibrado com estratégia mista ela convergirá para esta última. Segue uma síntese dos resultados de convergência discutidos até aqui.

Proposição 4. Se no estado estacionário com estratégia pura $\left.f\left(\tilde{k}_{s}\right)-\tilde{k}_{s} f^{\prime}\left(\tilde{k}_{s}\right)\right] R^{\lambda}>\omega$, então o ponto de equilíbrio $\left(\tilde{k}_{s}, 1\right) \in \Theta$ será um atrator local. Se $\left.f\left(\tilde{k}_{s}\right)-\tilde{k}_{s} f^{\prime}\left(\tilde{k}_{s}\right)\right] R^{\lambda}<\omega$, então o ponto de equilíbrio $\left(\tilde{k}_{s}, 1\right) \in \Theta$ será localmente instável, mais precisamente um ponto de sela cujo ramo estável será o locus $\left\{(k, \ell) \in \Theta: k \neq \tilde{k}_{s}\right.$ e $\left.\ell=1\right\}$. O estado estacionário com estratégia mista $\left(k^{*}, \ell^{*}\right) \in \Theta$ é um atrator local.

Prova: Apêndice 4.

Quando a economia doméstica na vizinhança do estado estacionário com estratégia pura apresenta um salário efetivo maior que o salário efetivo do resto do mundo, o

19 Fornecedora ou receptora líquida de mão-de-obra. 
sistema econômico apresenta duas bases locais de atração: uma em torno do estado estacionário com estratégia pura e outra em torno do estado estacionário com estratégia mista. Mais uma vez aparece no modelo a propriedade de path dependence, ou seja, a depender do estado inicial da economia doméstica ela poderá convergir para uma ou outra destas trajetórias de crescimento equilibrado.

\section{CONSIDERAÇÕES FINAIS}

Neste trabalho apresentou-se um modelo de crescimento econômico do tipo SolowSwan com migração. O fluxo migratório foi modelado como um jogo evolucionário, mais precisamente como uma dinâmica de seleção, a partir da hipótese de que os trabalhadores apresentam diferenças idiossincráticas com respeito às suas metas salariais.

Os resultados alcançados corroboram em parte os obtidos na abordagem tradicional. Ou seja, as características de longo prazo da economia doméstica dependem fundamentalmente do seu saldo migratório líquido no estado estacionário. Todavia, novos resultados foram obtidos. Na versão sem efeito congestionamento do modelo evolucionário a economia doméstica converge para o estado estacionário se absorver completamente a população do resto do mundo, o que acontece se ela apresentar nesse estado um salário efetivo superior ao do resto do mundo. Se a economia doméstica em seu estado estacionário apresentar um salário efetivo inferior ao do resto do mundo, toda a sua população acabará emigrando para esse último. $\mathrm{O}$ modelo evolucionário sem efeito congestionamento, portanto, põe em evidência a impossibilidade de existência da economia doméstica como um sistema distinto do resto do mundo, deduzida no modelo de referência sem efeito congestionamento. Em outras palavras, o modelo evolucionário sem congestionamento torna explícita, diferentemente do modelo de referência sem congestionamento, a impossibilidade de a economia doméstica trilhar sua trajetória de crescimento equilibrado como uma perpétua receptora ou fornecedora líquida de mão-de-obra.

No modelo de referência sem congestionamento infere-se a coexistência da economia doméstica e do resto do mundo somente se ocorrer a homogeneização dos salários efetivos entre essas duas regiões, quando a economia doméstica encontrar-se em seu estado estacionário. Essa mesma conclusão é extraída do modelo evolucionário sem congestionamento. Todavia, do modelo evolucionário é inferido um resultado adicional, a saber, o tamanho relativo da população da economia doméstica depende das condições iniciais dessa última. 
A possibilidade de extinção da economia doméstica ou do resto do mundo não é sustentada pelos fatos. Uma das causas desta predição é encontrada na hipótese implícita de que o crescimento (declínio) populacional acelerado pela imigração (emigração) líquida não acarreta um congestionamento (descongestionamento) dos fatores/recursos fixos da economia doméstica. A incorporação do efeito congestionamento ao modelo evolucionário não exclui a possibilidade de fusão da economia doméstica e do resto do mundo em uma única economia, caso a economia doméstica apresente um diferencial de salários efetivos atrativo em seu estado estacionário. Entretanto, a introdução do efeito congestionamento trouxe à tona a possibilidade de convergência para um estado estacionário com a existência simultânea da economia doméstica e do resto do mundo.

Para finalizar, cabe destacar que uma comparação mais precisa entre as predições do modelo de referência e as do modelo evolucionário poderia ser alcançada por meio da comparação das propriedades de convergência desse último com as respectivas propriedades de convergência do modelo de referência.

\section{APÊNDICE 1: PROVA DA PROPOSIÇÃO I}

Um estado de equilíbrio do sistema (3.1) é uma solução do seguinte sistema:

$$
\begin{array}{r}
s f(k)-\eta k-(1-\ell)(k-\kappa) \phi(k)=0 \\
\ell(1-\ell) \phi(k)=0
\end{array}
$$

Considerando a segunda equação do sistema (A.1) conclui-se que esta é satisfeita se $\ell=1$ ou $\phi(k)=0$. Na presente demonstração parte-se da premissa de que existe uma razão capital-trabalho efetivo $k_{c}$, definida implicitamente por (2.9), tal que o fluxo migratório líquido é nulo. Dada a existência de $k_{c}$ sua unicidade é conseqüência do fato de que $\frac{d}{d k}\left[f(k)-k f^{\prime}(k)\right]=-k f^{\prime \prime}(k)>0$, devido à premissa de rendimentos físicos marginais decrescentes e mercado de trabalho perfeitamente competitivo.

Existência do ponto de equilíbrio $\left(k_{s}, 1\right) \in \Theta$ :

Se $\ell=1$ então a primeira equação de (A.1) reduz-se a:

$$
s f(k)-\eta k=0
$$


a qual pode ser transformada para $k>\kappa>0 \mathrm{em}$ :

$$
\frac{s f(k)}{k}-\eta=0
$$

Portanto, basta provar que existe um valor $k_{s}>\kappa>0$ tal que (A.2a) é satisfeita. Usando a regra de L'Hôpital e o fato de que $\lim _{k \rightarrow \infty} f^{\prime}(k)=0$ (uma das condições de Inada) infere-se que $\lim _{k \rightarrow+\infty}[s f(k) / k]=0$ e, portanto:

$$
\lim _{k \rightarrow+\infty}\left[\frac{s f(k)}{k}-\eta\right]=-\eta<0
$$

Como, por hipótese:

$$
\frac{s f(\kappa)}{\kappa}-\eta>0
$$

e $f(k)$ é contínua em qualquer $k \geq 0$, infere-se, com base no teorema do valor intermediário, que existe um $k_{s}>\kappa>0$, tal que (A.2a) é satisfeita.

Unicidade do ponto de equilibrio $\left(k_{s}, 1\right) \in \Theta$ :

Desde que a produtividade física marginal do trabalho é estritamente positiva, isto é, $f(k)-k f^{\prime}(k)>0$, então:

$$
\frac{\partial}{\partial k}\left[\frac{s f(k)}{k}-\eta\right]=-\frac{s\left[f(k)-k f^{\prime}(k)\right]}{k^{2}}<0
$$

e, portanto, o lado esquerdo de (A.2a) é uma função estritamente decrescente de $k$. Logo, $k_{s}$ é único.

Existência de um continuum de equilibrios:

Para que $\phi(k)=0$ deve-se ter, considerando (2.9), $k=k_{c}$. Se $\phi(k)=0$, então a primeira equação de (A.l) reduz-se a:

$$
s f(k)-\eta k=0
$$


Esta última equação é satisfeita se, e somente se, $k=k_{s}$. Logo, a única maneira de ambas as condições serem satisfeitas é ter-se $k_{s}=k_{c}$. Se isto acontecer, a solução do sistema (A.l) independe do valor que a variável $\ell$ assume em $\Theta$. Portanto, todos os pontos pertencentes ao conjunto $\left\{(k, \ell) \in \Theta: k=k_{s}=k_{c}\right\}$ são pontos de equilíbrio do sistema (3.1).

\section{APÊNDICE 2: PROVA DA PROPOSIÇÃO 2}

PARTe 1: O equilibrio $\left(k_{s}, 1\right)$ églobalmente assintoticamente estável se $\left.f\left(k_{s}\right)-k_{s} f^{\prime}\left(k_{s}\right)\right]>\omega$.

A construção do diagrama de fases deste caso foi realizada na seção três. A análise dos autovalores da matriz Jacobiana da linearização em torno do estado estacionário $\left(k_{s}, 1\right)$ está de acordo com as conclusões qualitativas globais obtidas. A matriz Jacobiana da linearização em torno do estado estacionário $\left(k_{s}, 1\right)$ é dada por:

$$
J\left(k_{s}, 1\right)=\left[\begin{array}{c:c}
s f^{\prime}\left(k_{s}\right)-\eta & \left(k_{s}-\kappa\right) \phi\left(k_{s}\right) \\
\hdashline 0 & -\phi\left(k_{s}\right)
\end{array}\right]
$$

cujos autovalores são:

$$
\lambda_{1}=s f^{\prime}\left(k_{s}\right)-\eta<0 \text { e } \lambda_{2}=-\phi\left(k_{s}\right)
$$

O segundo autovalor é negativo se $k_{s}>k_{c}$. De (A.8) infere-se, então, que o estado estacionário $\left(k_{s}, 1\right)$ é um sorvedouro da linearização em sua vizinhança quando $k_{s}>k_{c}$, pois todos os autovalores associados possuem partes reais negativas. Assim, com base no teorema de Hartman-Grobman, conclui-se que o estado estacionário $\left(k_{s}, 1\right)$ é um atrator local se $k_{s}>k_{c}$ (cf. Figura 2a).

Parte 2: O equilíbrio $\left(k_{s}, 1\right) \in \Theta$ é instável se $\left.f\left(k_{s}\right)-k_{s} f^{\prime}\left(k_{s}\right)\right]<\omega$ e $\quad \ell \rightarrow 0^{+}$se $\left(\ell_{0}, k_{0}\right) \in \operatorname{int} \Theta$. 
Posicionamento da curva de demarcação $k$ no plano de fases:

Suponha $k_{s}<k_{c}$ (cf. Figura 2b). Considerando (3.3), para $k=k_{s}<k_{c}$ tem-se $\psi\left(k_{s}\right)=0 \mathrm{e}$, portanto, $\ell=1$. Logo, o ponto $\left(k_{s}, 1\right)$ pertence à curva de demarcação $k$. Suponha $0<\ell \leq 1$. Para qualquer $k \in\left(\kappa, k_{s}\right]$ tem-se $s f(k)-\eta k>0$ e $\phi(k)<0$, $\operatorname{logo} \dot{k}=s f(k)-\eta k-(1-\ell)(k-\kappa) \phi(k)>0$. Para qualquer $k \in\left(k_{s}, \infty\right)$ tem-se $s f(k)-\eta k<0$ e $\phi(k)>0, \operatorname{logo} \dot{k}=s f(k)-\eta k-(1-\ell)(k-\kappa) \phi(k)<0$. Portanto, a curva de demarcação $k$ é um subconjunto do subespaço $\left\{(k, \ell) \in \Theta: k_{c}<k \leq k_{s}\right\}$.

\section{Inclinação da curva de demarcação $k$ :}

A inclinação da curva de demarcação $k$ no subespaço $\left\{(k, \ell) \in \Theta: k_{c}<k \leq k_{s}\right\}$ é dada por (3.4). No intervalo $\left(k_{s}, k_{c}\right)$ tem-se $f(k)-\eta k<0, k-\kappa>0$ e $\phi(k)<0$, porém a expressão $s f^{\prime}(k)-\eta$ apresenta sinal indeterminado. Logo, a função (3.4) pode não ser monótona no intervalo $\left(k_{s}, k_{c}\right)$. A função (3.4) apresenta o seguinte comportamento na fronteira do intervalo $\left[k_{s}, k_{c}\right)$ :

$$
\begin{aligned}
& \lim _{k \rightarrow k_{s}^{+}} \psi(k)=\frac{\lim _{k \rightarrow k_{s}^{+}} s f(k)-\eta k}{\left(k_{s}-\kappa\right) \phi\left(k_{s}\right)}=\frac{0}{\left(k_{s}-\kappa\right) \phi\left(k_{s}\right)}=0 \\
& \lim _{k \rightarrow k_{c}^{-}} \psi(k)=\left[\frac{s f\left(k_{c}\right)-\eta k_{c}}{k_{c}-\kappa}\right] \lim _{k \rightarrow k_{c}^{-}} \frac{1}{\phi(k)}=\left[\frac{s f\left(k_{c}\right)-\eta k_{c}}{k_{c}-\kappa}\right] \cdot(-\infty)=\infty
\end{aligned}
$$

Pelo teorema do valor intermediário, então, infere-se que existe um $k_{w}$, tal que $\psi\left(k_{w}\right)=1$. Suponha que $k_{w}$ é único. Como $k_{s}<k_{c}$, então:

$$
\psi\left(k_{s}\right)=\frac{\eta-s f^{\prime}\left(k_{s}\right)}{\left(k_{s}-\kappa\right) \phi\left(k_{s}\right)}<0
$$

Portanto, deve-se ter $\psi^{\prime}(k)<0$ em todo $k \in\left(k_{w}-\varepsilon, k_{w}\right)$ para um $\varepsilon>0$ suficientemente pequeno, pois do contrário $k_{w}$ não seria único.

Considerando (A.10), em uma vizinhança suficientemente pequena de $k_{s}$ a curva de demarcação $k$ é também negativamente inclinada. Portanto, a curva de demarcação $k$ pode apresentar uma reversão de inclinação somente no interior do intervalo $\left[k_{s}, k_{w}\right)$, 
como ilustrado na Figura 2b. Se isso ocorre ou não, dada a unicidade do ponto $\left(k_{w}, 0\right)$, é irrelevante para as conclusões qualitativas sobre a dinâmica do sistema.

Determinação da configuração das linhas direcionais do campo vetorial:

No intervalo $\left(k_{s}, k_{c}\right)$ tem-se:

$$
\left.\frac{\partial \dot{k}}{\partial \ell}=(k-\kappa)\right] \phi(k)<0
$$

portanto, pontos localizados na região $\left\{(k, \ell) \in \Theta: k_{s}<k \leq k_{c}\right\}$ e abaixo (acima) da curva de demarcação $k$ apresentam $\dot{k}<0(\dot{k}>0)$. Isso completa a construção do diagrama de fase da Figura $2 b$.

Análise de estabilidade local via linearização:

Considerando (A.7) e (A.8), infere-se que o estado estacionário $\left(k_{s}, 1\right)$ é um ponto de sela da linearização em sua vizinhança quando $k_{s}<k_{c}$, já que os autovalores associados possuem partes reais com sinais opostos. Assim, com base no teorema de Hartman-Grobman, conclui-se que o estado estacionário $\left(k_{s}, 1\right)$ é um ponto de sela local se $k_{s}<k_{c}$ (cf. Figura $2 \mathrm{~b}$ ).

PARTE 3: O subespaço $\left\{(k, \ell) \in \Theta: \ell<1\right.$ e $\left.k_{c}<k \leq k_{s}\right\}$ é um atrator global se $f\left(k_{s}\right)-k_{s} f^{\prime}\left(k_{s}\right)=\omega$.

Suponha $k_{s}=k_{c}$. Neste caso, considerando a primeira equação em (3.1), a isoclina $\dot{k}=0$ é o locus $\left\{(k, \ell) \in \Theta: k=k_{s}=k_{c}\right\}$. Assim, para qualquer $k<k_{s}=k_{c}$ tem-se $s f(k)-\eta k>0$ e $\phi(k)<0, \log$ o $\dot{k}=s f(k)-\eta k-(1-\ell)(k-\kappa) \phi(k)>0$ e $\dot{\ell}=\ell(1-\ell) \phi(k)<0$. Para qualquer $k>k_{s}=k_{c}$ tem-se $s f(k)-\eta k<0$ e $\phi(k)>0$, então $\dot{k}<0$ e $\dot{\ell}>0$. Essa combinação das setas direcionais (cf. Figura 2c) demonstra que o subespaço $\left\{(k, \ell) \in \Theta: \ell<1\right.$ e $\left.k_{c}<k \leq k_{s}\right\}$ é um atrator global. 


\section{APÊNDICE 3: PROVA DA PROPOSIÇÃO 3}

Um estado de equilíbrio de (4.3) é uma solução do seguinte sistema:

$$
\begin{aligned}
& s f(k)(R / \ell)^{\lambda}-\eta k-(1-\ell)(k-\kappa) \varphi(k, \ell)=0 \\
& \ell(1-\ell) \varphi(k, \ell)=0
\end{aligned}
$$

Desde que $\ell>0$, infere-se que a segunda equação do sistema (A.12) é satisfeita se $\ell=1$ ou $\left[f(k)-k f^{\prime}(k)\right](\bar{R} / \ell)^{\lambda}=\omega$, tal que $\varphi(k, \ell)=G\left(\left[f(k)-k f^{\prime}(k)\right](R / \ell)^{\lambda}\right)-G(\omega)=0$.

Existência do ponto de equilíbrio de estratégia pura $\left(\tilde{k}_{s}, 0\right)$ :

Se $\ell=1$, então a primeira equação em (A.12) reduz-se a:

$$
s f(k) R^{\lambda}-\eta k=0
$$

a qual pode ser transformada para $k>\kappa>0 \mathrm{em}$ :

$$
\frac{s f(k) R^{\lambda}}{k}-\eta=0
$$

Portanto, basta provar que existe um valor $\tilde{k}_{s}>\kappa>0$, tal que (A.13a) é satisfeita. Usando a regra de L'Hôpital e o fato de que $\lim _{k \rightarrow \infty} f^{\prime}(k)=0$ (uma das condições de Inada), infere-se que $\lim _{k \rightarrow+\infty}\left[s f(k) R^{\lambda} / k\right]=0$ e, portanto:

$$
\lim _{k \rightarrow+\infty}\left[\frac{s f(k) R^{\lambda}}{k}-\eta\right]=-\eta<0
$$

Como, por hipótese:

$$
\frac{s f(\kappa) R^{\lambda}}{\kappa}-\eta>0
$$

e $f(k)$ é contínua em qualquer $k \geq 0$, infere-se, com base no teorema do valor intermediário, que existe um $\tilde{k}_{s}>\kappa>0$, tal que (A.13a) é satisfeita. 
Unicidade do ponto de equilibrio de estratégia pura $\left(\tilde{k}_{s}, 0\right)$ :

Desde que a produtividade física marginal do trabalho efetivo é estritamente positiva, isto é, $\left[f(k)-k f^{\prime}(k)\right](R / \ell)^{\lambda}>0$, então:

$$
\frac{\partial}{\partial k}\left[\frac{s f(k) R^{\lambda}}{k}-\eta\right]=-\frac{s R^{\lambda}\left[f(k)-k f^{\prime}(k)\right]}{k^{2}}<0
$$

e, portanto, o lado esquerdo de (A.13a) é uma função estritamente decrescente de $k$. Logo, $\tilde{k}_{s}$ é único.

Existência do ponto de equilibrio de estratégia mista $\left(k^{*}, \ell^{*}\right)$ :

Se $0<\ell<1$, então a segunda equação de (A.12) é satisfeita se, e somente se, $\left[f(k)-k f^{\prime}(k)\right](R / \ell)^{\lambda}=\omega$, a partir da qual obtém-se:

$$
\ell=R\left[\frac{f(k)-k f^{\prime}(k)}{\omega}\right]^{1 / \lambda}
$$

Introduzindo (A.17) na primeira equação de (A.12) resulta:

$$
\mu(k) \equiv s \frac{f(k)}{k}-\eta\left[\frac{f(k)-k f^{\prime}(k)}{\omega}\right]=0
$$

Portanto, basta provar que existe um valor $k^{*}>\kappa>0$ tal que (A.18) é satisfeita. Seja $k_{c}$ o valor definido implicitamente por (2.9). Desde que $k_{c}>\kappa>0$, sabe-se que:

$$
\lim _{k \rightarrow k_{c}^{+}} \mu(k)=\frac{s f\left(k_{c}\right)}{k_{c}}>0
$$

Usando a regra de L'Hôpital e o fato de que $\lim _{k \rightarrow \infty} f^{\prime}(k)=0$ (uma das condições de Inada) infere-se que:

$$
\lim _{k \rightarrow \infty}[f(k) / k]=0
$$

Além disso, dado que $\frac{d}{d k}\left[f(k)-k f^{\prime}(k)\right]=-k f^{\prime \prime}(k)>0$, decorre que o salário doméstico efetivo é uma função estritamente crescente da razão capital-trabalho efetivo e, portanto: 


$$
\lim _{k \rightarrow \infty}\left[f(k)-k f^{\prime}(k)\right]=\infty
$$

Os limites (A.20) e (A.2l) permitem concluir que:

$$
\lim _{k \rightarrow \infty} \mu(k)=\lim _{k \rightarrow \infty}\left\{\frac{s f(k)}{k}-\eta\left[\frac{f(k)-k f^{\prime}(k)}{\omega}\right]\right\}=-\infty
$$

Como $f(k)$ é uma função $C^{1}$ em qualquer $k \geq 0$, a partir de (A.19) e (A.22) inferese, baseado no teorema do valor intermediário, que existe um $k^{*}>k_{c}>\kappa$, tal que vale (4.6). Sendo assim, por (A.17) existe para cada $k^{*}$ um único valor bem definido de $\ell^{*}>0$ dado por (4.5). Considerando (4.5), para que se tenha $\ell^{*}<1$ a seguinte condição deve ser satisfeita:

$$
f\left(k^{*}\right)-k^{*} f^{\prime}\left(k^{*}\right) R^{\lambda}<\omega
$$

Unicidade do ponto de equilibrio de estratégia mista $\left(k^{*}, \ell^{*}\right)$ :

Desde que $f(k)-k f^{\prime}(k)>0$ e $f^{\prime \prime}(k)<0$, então:

$$
\mu(k)=-\frac{s\left[f(k)-k f^{\prime}(k)\right]}{k^{2}}+\frac{\eta k f^{\prime \prime}(k)}{\omega}<0
$$

e, portanto, $\mu(k)$ é uma função estritamente decrescente de $k$. Logo, $k^{*}$ é único, conseqüentemente, considerando (4.5), o ponto $\left(\ell^{*}, k^{*}\right)$ é único.

\section{APÊNDICE 4: PROVA DA PROPOSIÇÃO 4}

A matriz Jacobiana da linearização em torno do estado estacionário $\left(\tilde{k}_{s}, 1\right)$ é dada por:

$$
J\left(\tilde{k}_{s}, 1\right)=\left[\begin{array}{c:c}
s f^{\prime}\left(\tilde{k}_{s}\right) R^{\lambda}-\eta & \left(\tilde{k}_{s}-\kappa\right) \varphi\left(\tilde{k}_{s}, 1\right)-\lambda s f\left(\tilde{k}_{s}\right) R^{\lambda} \\
\hdashline 0 & -\varphi\left(\tilde{k}_{s}, 1\right)
\end{array}\right]
$$

cujos autovalores são:

$$
\lambda_{1}=s f^{\prime}\left(\tilde{k}_{s}\right) R^{\lambda}-\eta-\varphi\left(\tilde{k}_{s}, 1\right) \text { e } \lambda_{2}=-\varphi\left(\tilde{k}_{s}, 1\right)
$$


Dado (A.26), desde que $s f^{\prime}\left(\tilde{k}_{s}\right) R^{\lambda}-\eta<0$, conclui-se que o ponto de equilíbrio $\left(\tilde{k}_{s}, 1\right)$ será um sorvedouro da linearização se $\left.f\left(\tilde{k}_{s}\right)-\tilde{k}_{s} f^{\prime}\left(\tilde{k}_{s}\right)\right] R^{\lambda}>\omega$, pois sob tal condição $\varphi\left(\tilde{k}_{s}, 1\right)>0$ e, portanto, ambos os autovalores são estritamente negativos. Então, pelo teorema de Hartman-Grobman conclui-se que o ponto de equilíbrio $\left(\tilde{k}_{s}, 1\right)$ do sistema (4.3) é um atrator local se $\left.f\left(\tilde{k}_{s}\right)-\tilde{k}_{s} f^{\prime}\left(\tilde{k}_{s}\right)\right] R^{\lambda}>\omega$. Quando a economia doméstica é receptora líquida de mão-de-obra no estado estacionário em questão, isto é, se $\left.f\left(\tilde{k}_{s}\right)-\tilde{k}_{s} f^{\prime}\left(\tilde{k}_{s}\right)\right] R^{\lambda}>\omega$, então o ponto de equilíbrio $\left(\tilde{k}_{s}, 1\right)$ será um ponto de sela da linearização em sua vizinhança, já que os autovalores apresentam sinais opostos. $\mathrm{O}$ ramo estável será o locus $\left\{(\ell, k) \in \Theta: \ell=1\right.$ e $\left.k \neq \tilde{k}_{s}\right\}$. Conseqüentemente, pelo teorema de Hartman-Grobman, conclui-se que o ponto de equilíbrio $\left(\tilde{k}_{s}, 1\right)$ do sistema $(4.3)$ é um repulsor em uma vizinhança deste ponto contida no conjunto $\left\{(k, \ell) \in \Theta: k \neq \tilde{k}_{s}\right.$ e $\left.\ell<1\right\}$ se $\left.f\left(\tilde{k}_{s}\right)-\tilde{k}_{s} f^{\prime}\left(\tilde{k}_{s}\right)\right] R^{\lambda}<\omega$.

A matriz Jacobiana da linearização em torno do estado estacionário $\left(k^{*}, \ell^{*}\right)$ é dada por:

$$
J\left(k^{*}, \ell^{*}\right)=\left[\begin{array}{c:c}
s f^{\prime}\left(k^{*}\right)\left(R / \ell^{*}\right)^{\lambda}-\eta-\left(1-\ell^{*}\right)\left(k^{*}-\kappa\right) \varphi_{k}^{*} \mid-s f\left(k^{*}\right) \lambda\left(R / \ell^{*}\right)^{\lambda} / \ell^{*}-\left(1-\ell^{*}\right)\left(k^{*}-\kappa\right) \varphi_{\ell}^{*} \\
\hdashline \ell^{*}\left(1-\ell^{*}\right) \varphi_{k}^{*} & \vdots
\end{array}\right]
$$

na qual

$$
\begin{aligned}
& \varphi_{k}^{*} \equiv \frac{\partial \varphi\left(k^{*}, \ell^{*}\right)}{\partial k}=-G^{\prime}(\cdot) k^{*} f^{\prime \prime}\left(k^{*}\right)\left(\frac{R}{\ell^{*}}\right)^{\lambda}>0 \text { e } \\
& \varphi_{k}^{*} \equiv \frac{\partial \varphi\left(k^{*}, \ell^{*}\right)}{\partial k}=-G^{\prime}(\cdot) k^{*}\left[f\left(k^{*}-k^{*} f^{\prime}\left(k^{*}\right)\right]\left(\frac{R}{\ell^{*}}\right)^{\lambda} \frac{\lambda}{\ell^{*}}<0 .\right.
\end{aligned}
$$

Da matriz Jacobiana (A.27) obtém-se:

$$
\operatorname{det} J\left(k^{*}, \ell^{*}\right)=\left(1-\ell^{*}\right)\left\{s f\left(k^{*}\right)\left(\frac{R}{\ell^{*}}\right)^{\lambda} \lambda \varphi_{k}^{*}+\ell^{*}\left[s f^{\prime}\left(k^{*}\right)\left(\frac{R}{\ell^{*}}\right)^{\lambda}-\eta\right] \varphi_{\ell}^{*}\right\}>0
$$

$\mathrm{e}$

$$
\operatorname{trJ}\left(k^{*}, \ell^{*}\right)=s f^{\prime}\left(k^{*}\right)\left(\frac{R}{\ell^{*}}\right)^{\lambda}-\eta-\left(1-\ell^{*}\right)\left(k^{*}-\kappa\right) \varphi_{k}^{*}+\ell^{*}\left(1-\ell^{*}\right) \varphi_{\ell}^{*}<0
$$


De (A.28) infere-se que o ponto de equilíbrio $\left(k^{*}, \ell^{*}\right)$ é um sorvedouro da linearização em sua vizinhança. Pelo teorema de Hartman-Grobman, o ponto de equilíbrio $\left(k^{*}, \ell^{*}\right)$ do sistema $(4.3)$ é um atrator local.

\section{REFERÊNCIAS BIBLIOGRÁFICAS}

Barro, R. J.; Sala-i-Martin, X. Economic growth. Cambridge: MIT Press, 2001.

Bonomo, M.; Carrasco, V.; Moreira, H. Aprendizado evolucionário, inércia inflacionária e recessão em desinflaçóes monetárias. Revista Brasileira de Economia, v. 57 , n. 4 , out.-dez. 2003.

Hofbauer, J.; Sigmund, K. Evolutionary games and population dynamics. Cambridge: Cambridge University Press, 1998.

Ponti, G. Continuous-time evolutionary dynamics: theory and pratice. Research in Economics, v. 54, p. 187-214, 2002.

Prado, E. F. S. Dilema dos prisioneiros e dinâmicas evolucionárias. Estudos Econômicos, v. 29 , n. 2 , p. 249-266, abr.-jun. 1999.

. Dois modelos clássicos de economia monetária. Economia Aplicada, v. 5, n. 3, p. 547-567, jul.-set. 2001.

Prado, E. F. S.; Kadota, D. K.; Soromenho, J. E. C. Survival of technologies: an evolutionary game approach. Economia Aplicada, v. 7, n. 2, abr.-jun. 2003.

Samuelson, L. Evolutionary games and equilibrium selection. Cambridge: MIT Press, 1997.

Silveira, J. J. Ciclos clássicos num ambiente de racionalidade limitada. Estudos Econômi$\cos$, v. 33, n. 4, p. 701-734, out.-dez. 2003.

Silveira, J. J.; Sanson, J. R. The Harris-Todaro labor allocation mechanism as an evolutionary game. In: Latin American Meeting of the Econometric Society, 2004, Santiago. Anais do Latin American Meeting of the Econometric Society: Santiago: LAMES, 2004, I CD ROOM.

Simon, H. A. Satisficing. In: Eatwell, J.; Milgate, M.; Newman, P (eds.), The New Palgrave: a dictionary of economics. London: MacMillan Press, 1987.

Soromenho, J. E. C.; Kadota, D. K.; Prado, E. F. S. Scale and externalities in an evolutionary game model. Estudos Econômicos, v. 31, n. 3, p. 529-550, jul.-set. 2001 .

; Silveira, J. J. Demanda e desemprego no médio prazo. São Paulo: IPE-USP, 2006. Texto para Discussão apresentado no Programa de Seminários Complex. Dísponível em: <http://www.fea.usp.br/>. Acesso em: 1 fev. 2006. 
Vega-Redondo, F. Evolution, games and economic behaviour. Oxford: Oxford University Press, 1996.

Weibull, J. W. Evolutionary game theory. Cambridge: MIT Press, 1995. 